\title{
An Essential Service Decision Model for ABA Providers During Crisis
}

\author{
Richard A. Colombo ${ }^{1} \cdot$ Michele Wallace $^{1} \cdot$ Rachel Taylor $^{1}$ \\ Published online: 22 May 2020 \\ (C) Association for Behavior Analysis International 2020
}

\begin{abstract}
In the United States, applied behavior analysis (ABA) is broadly recognized as a medically necessary treatment for individuals diagnosed with autism and related disorders (Association of Professional Behavior Analysts, 2020, Guidelines for practicing applied behavior analysis during COVID-19 pandemic, Retrieved from https://cdn.ymaws.com/www.apbahome.net/resource/ collection/1FDDBDD2-5CAF-4B2A-AB3F-DAE5E72111BF/APBA_Guidelines_-_Practicing_During_COVID-19_ Pandemic_040920.pdf). We argue that this designation should not be called into question in light of a particular disaster and that it is critical to consider that an interruption of services can have long-lasting effects on the treatment of the individual (practitioners are ethically obligated to uphold the continuity of services while doing no harm). This dilemma might be ameliorated by a decision model that considers the prioritization of immediate needs, the vulnerability of clients, and the competency of service providers. Just as the medical field prioritizes immediate needs during crisis situations and defers routine appointments (e.g., physicals, checkups), the ABA field can make similar evidence-based decisions. The purpose of the current article is to provide a decision model for $\mathrm{ABA}$ practitioners who find themselves questioning the need for essential service delivery during the current COVID-19 pandemic. The impact of this model goes beyond the needs of this crisis and can be applied to any emergency situation where services are at risk of interruption.
\end{abstract}

Keywords autism $\cdot$ COVID-19 $\cdot$ decision making $\cdot$ essential services $\cdot$ ethics $\cdot$ pandemic

Practitioners in the field of applied behavior analysis (ABA) are currently faced with crucial decisions about services for vulnerable populations. News about the coronavirus disease (COVID-19) increases daily, and a growing number of states are imposing "shelter-at-home" policies. As such, many practitioners will be faced with the challenging decision whether to maintain, modify, cease, or refer services or modify the treatment-delivery format. Many ethical standards apply to such situations: for example, that behavior analysts should make these decisions in the best interest of the client (Code 2.15[a]; Behavior Analyst Certification Board [BACB], 2014) and that a reasonable and timely process is undertaken for the continuation of services during an emergency (Code 2.15[b]; $B A C B, 2014)$. No previous research, of which we are aware, has evaluated the potentially devastating long-term impacts on child development of interrupting medically necessary

Richard A. Colombo

rcolombo@centerforaba.com

1 Center for Applied Behavior Analysis, 11150 W. Olympic Blvd, Los Angeles, CA 90064, USA services. In the absence of such research, the ABA practitioner is left to consider how an interruption of services (e.g., utilizing a different treatment-delivery model, modifying it, ceasing it) can potentially affect the life of an individual for many years, and many credentialed practitioners have turned to the $\mathrm{BACB}$ for guidance. The BACB has provided useful resources to help guide ABA practitioners (e.g., BACB, 2020), but ultimately agency directors and individual Board Certified Behavior Analysts will have to make decisions that can impact the health and well-being of their staff, clients, caregivers, and society. These decisions are compounded by the fact that $\mathrm{ABA}$ is considered a medically necessary treatment for individuals diagnosed with autism and related disorders (Association of Professional Behavior Analysts, 2020). This designation has afforded families living with autism many benefits (e.g., insurance funding for their treatment) and must be protected by every ABA practitioner who delivers these treatment services. The actions taken in this time of crisis might influence future discussions regarding $\mathrm{ABA}$, its professionalism, and its continued funding.

The weight of such an issue has inspired some to provide tools to practitioners who are considering the risk of continuing to provide in-person ABA treatment. Cox, Plavnick, and 
Brodhead (in press) supplied a risk assessment that attempts to address the associated dangers of both maintaining and interrupting in-person services. They offer several contributions to the literature addressing significant concerns related to the current COVID-19 crisis and related considerations for the provision of $\mathrm{ABA}$ services. Cox et al. recognize the potential harm of service interruption and the need to maintain medically necessary ABA treatment despite a given crisis. The authors also identify the potential harm caused by maintaining in-person, precrisis treatment-delivery models. As such, the authors recommend using an individualized, case-by-case approach (as opposed to "blanketed" responses) with ongoing risk assessments while adhering to ethical obligations, especially during this time of crisis. The recommendation to consider each client individually and avoid "knee-jerk" responses of either continuing or discontinuing in-person services for all clients one serves is a crucially important contribution. However, the complexity of this topic requires more tools to help practitioners navigate these challenging and high-stakes decisions.

In this article, we offer several discussion points to elaborate on the conversation that Cox et al. began. In particular, the authors suggest that some practitioners may be inaccurately defining in-person ABA service provision as "essential." Specifically, although the authors acknowledge that essential services during a crisis are in fact defined by local, state, and federal governing authorities, they also suggest that appropriate risk assessment outcomes will likely define in-person ABA services as nonessential for the majority of current consumers. This suggestion may at first blush seem to conflict with the broad agreement that ABA is a medically necessary treatment for autism. However, the authors argued that inperson ABA may not be essential during a time of global pandemic and that this ethical decision needs to be made at the level of each individual client. This subtle distinction is important and is likely easy to misconstrue. If this position is misunderstood as arguing that ABA is a nonessential service, it may have both immediate and long-term impacts on local, state, and federal support for ABA (especially given the other uncontested employment titles that are listed as essential; e.g., speech-language pathologists and occupational therapists). We argue that it is critically important to adhere to the position that behavioral health services (including ABA) are essential and, per this established definition, to now consider the need for continued in-person delivery of these services. We are in a time of crisis that by definition increases the likelihood of significantly decreased resources (e.g., due to individual essential workers' personal choices, established fundingprovider safety precautions). Overall, we propose that the question should not be whether ABA is essential; the question should be, how can we protect continuity of care during a time of crisis with decreased in-person direct support from essential workers? Although most behavior analysts view the designation of ABA as medically necessary as crucial for our clients to receive effective treatment and as central to the continued health of our profession, it would be unwise to think the field is immune to negative legal or policy changes. For example, Keenan et al. (2015) described the vast difference between ABA services in the United States and Europe. Specifically, the authors note that the United Kingdom has adopted eclectic modalities, even after ABA had previously been in relatively good standing. Misconceptions spread through academic, professional, and political circles without correction from informed behavior analysts. Although Keenan et al. speculate that this is unlikely in the United States, there may still be ways in which ABA can lose its broad-based support. One manner in which ABA might come under scrutiny is through the profession's response during a time of crisis. For example, if ABA practitioners interrupt services without showing efforts to maintain continuity of care, funding providers may be more likely to reevaluate the extent to which ABA service provision is considered to be necessary. Thus, it is imperative to equip practitioners with effective strategies for initial and ongoing risk assessments to regularly evaluate whether the safety of staff, clients, caregivers, and society can be reasonably protected.

\section{A Decision Model for Service Interruption During a Time of Crisis}

The purpose of the current article is to offer a crisis decision model for practitioners who are confronted with having to mitigate potential health and safety risks while protecting continuity of care during a crisis. What follows is an attempt at constructing a more comprehensive crisis-informed decision model, including a structured process to modify or interrupt a portion of services as needed. Although this decision model was inspired by the impact of COVID-19, it was developed as a resource that may be generalizable to any crisis situation (e.g., natural disasters) by emphasizing data-based decision making.

Figure 1 illustrates the decision model and its five levels of consideration. The model assumes that caregivers and funding providers have independently requested and provided informed consent for ongoing treatment; thus, this is not a question within the model. If at any point, caregivers or funding providers indicate that an ABA provider's services are no longer desired or necessary, the ABA provider must document this decision and set up periodic check-ins for the potential reinstatement of services.

\section{Decision Level 1: The Physical Setting}

The first level of consideration is in regard to the physical location of the services (e.g., home, school, center). During 


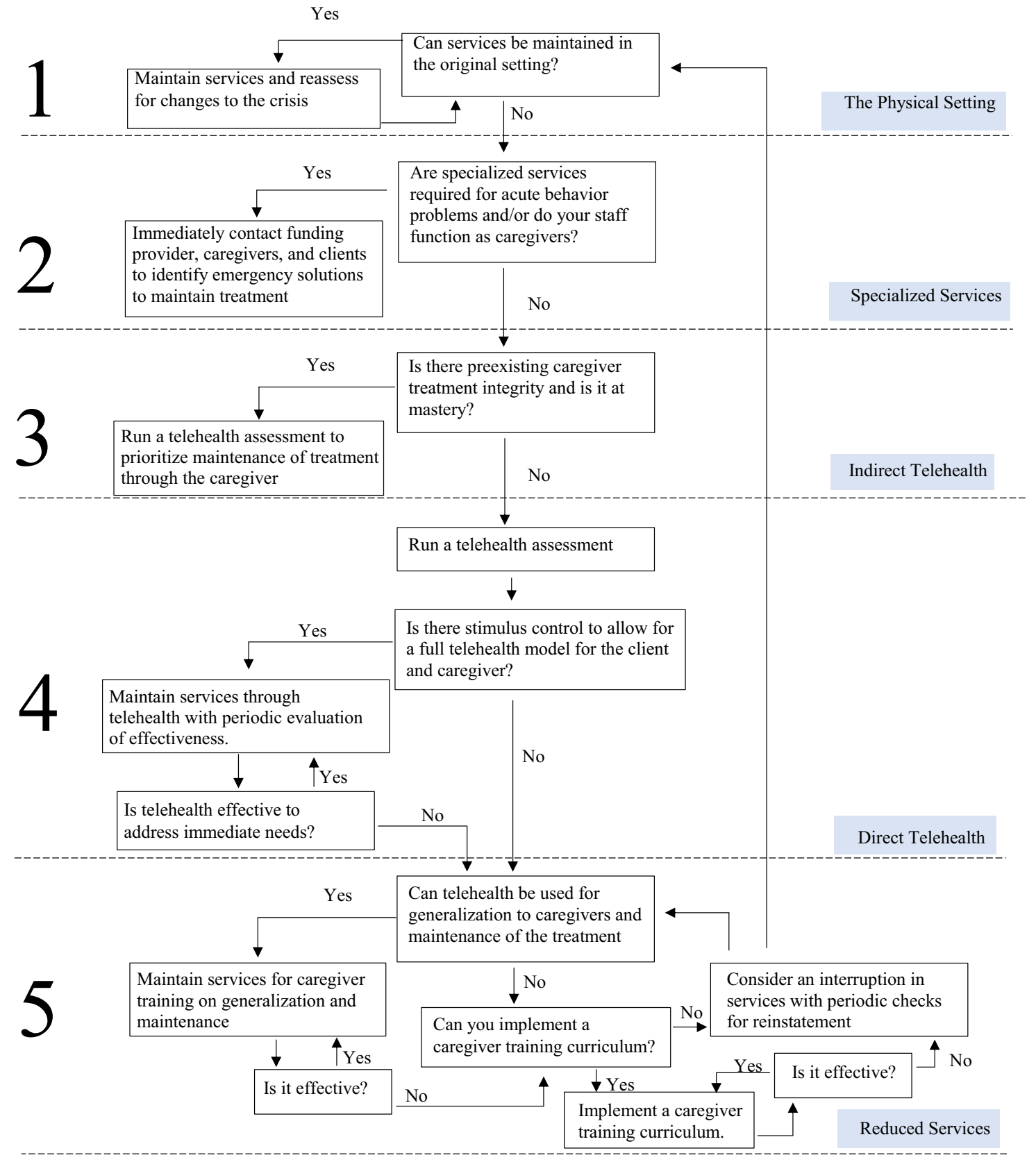

Figure 1. Crisis decision model.

or after a crisis, there may be physical barriers (e.g., flooding) or political mandates (e.g., government orders) that prevent direct, in-person treatment. The model begins by asking whether services can be maintained in the original setting. If the answer is yes, practitioners should proceed with services as usual and reassess the safety of maintaining in-person services daily (or more frequently if warranted by the crisis). Minor changes might be appropriate and have little effect on the treatment implementation. Examples of these modifications include additional time to wash hands and disinfect surfaces and materials at the beginning and end of a shift (in the case of a pandemic) and working with the client at an alternative location (in the case of damage to the client's home after a natural disaster). If the service setting is preservedeven partially - services should remain as is. If services cannot be maintained in the original setting (i.e., the answer is no in the decision model), consider the second level of the decision model.

\section{Decision Level 2: Specialized Services}

Some ABA practitioners provide services to individuals whereby, if their treatment was interrupted, problem behavior is likely to result in hospitalization or incarceration due to its 
severity. This level also applies to practitioners who function as caregivers to clients living in residential facilities. Clients who are in this category might engage in a high frequency of intense physical aggression, self-injurious behavior, property destruction, or elopement. If practitioners answer yes to the second level of this model, they will need to act quickly. Providers will need to immediately contact caregivers and funding sources to determine the best course of action. Interruption at this level can lead to intrusive interventions (e.g., medical sedation, restraint, seclusion), risk of severe injury, and even death. Funding sources and state agencies (e.g., California's Department of Developmental Services) might be able to provide resources for the continuity of services (e.g., a location to house and maintain treatment) or offer exceptions to government orders (e.g., allowing staff to work during a pandemic and to be classified as essential workers).

\section{Decision Level 3: Indirect Telehealth}

The third level of consideration addresses situations in which severe problem behavior is not an issue or has already been eliminated but there is a question as to whether the caregiver can maintain the treatment plan. The model poses the following question: "Is there preexisting caregiver treatment integrity and is it at mastery?" Practitioners will answer yes if preexisting treatment integrity data show mastery of fundamental aspects of the treatment plan. These might include, but are not limited to, antecedent interventions, contingencies of reinforcement for replacement behavior, consequence interventions, and pertinent skill-acquisition targets (e.g., discrimination training, verbal behavior, listener behavior). If these conditions are met, the model recommends a telehealth assessment (e.g., Rodriguez, in press) to target the maintenance and development of the treatment plan through caregiver training. As the treatment modality changes and requires more from the caregivers, practitioners should prioritize maintenance and development to minimize errors of commission.

Fryling, Wallace, and Yassine (2012) reviewed the literature on treatment integrity and found that errors of commission (i.e., implementing the intervention erroneously) tend to have a greater impact on the outcome of treatment than errors of omission (i.e., failing to implement the intervention altogether). During caregiver training, practitioners may be able to avoid errors of commission by discontinuing or modifying interventions that are too complex or cumbersome for a caregiver. Reducing the intricacies of the case might mean that the treatment focuses on maintenance training rather than producing new treatment gains.

Research suggests that maintenance training is a necessary component of treatment if the behavior outcomes are to remain well beyond the cessation of services (Pennington, Simacek, McComas, McMaster, \& Elmquist, 2019). Even when maintenance training is used in research, some outcomes show a degree of regression in as soon as 2 weeks. If a crisis occurs before practitioners begin to thin the schedule of reinforcement and fade supplementary antecedent stimuli (i.e., maintenance training), behavior may regress quickly and problem behaviors could resurge or be shaped into more severe forms. Maintenance training might be the best decision within Level 3 depending on the circumstances. Conducting a telehealth assessment (e.g., Rodriguez, in press) will help with prioritizing treatment goals and potentially focusing on maintenance training. Pollard, Karimi, and Ficcaglia (2017) outline several ethical considerations for designing and implementing telehealth services.

If the answer is no to the third level of the model (i.e., no treatment integrity exists), move to the fourth level. This portion of the model focuses on transitioning to full telehealth for the caregiver and client.

\section{Decision Level 4: Direct Telehealth}

The fourth level of consideration is focused on the assessment and potential implementation of a full and direct telehealth modality. This decision model holds no assumption that telehealth is appropriate for all individuals. Arbitrarily applying telehealth without an operationalized plan might result in treatment regression, the creation of misconceptions (e.g., "ABA and telehealth don't work."), and the potential breeding of mistrust with funding providers. The Council of Autism Service Providers (2020) cautions practitioners to assess individuals for their eligibility for telehealth. They recommend that organizations develop an admission process to determine clinical appropriateness. As such, clients should be assessed on an individualized basis just as in any other service modality that employs ABA. Telehealth trainings will provide information to practitioners who will need to assess the appropriateness of the model. This model does not provide a comprehensive assessment of individuals' eligibility for telehealth. Instead, it advises careful deliberation.

Once a telehealth assessment is conducted to evaluate eligibility, the fourth level of the model asks whether there is stimulus control between the client and caregiver. If caregivers are able to maintain the learning setting with existing stimulus control, behavior technicians (BTs) may be able to use a telehealth model to maintain the treatment with the assistance of the caregiver. The BT would follow the treatment plans prescribed by the behavior analyst, who develops and modifies the programming as needed. If the caregiver has little or no stimulus control to maintain the learning setting, it is recommended that practitioners begin caregiver training to maintain the treatment plan and periodically evaluate effectiveness. If ongoing data collection continues to show positive 
outcomes, it is recommended that the caregiver-led telehealth be maintained as necessary. If the treatment integrity data show problems with caregiver-led telehealth (i.e., it is not effective), move to Level 5.

\section{Decision Level 5: Reduced Services}

The final level of the decision model pertains to the reduction of services. It starts with the qualifier that neither the BT via telehealth nor the caregiver has the stimulus control necessary to maintain the treatment effectively and safely. As such, an assessment is needed to see if the caregiver can be trained to gain a degree of stimulus control to maintain the progress of the client. If previously existing data indicate that this is possible (the answer is yes), a telehealth model should be used to (a) promote the development of stimulus control and (b) maintain the treatment outcomes. If data indicate effectiveness, maintain the treatment and reassess for a full telehealth model in Level 4. If maintenance training is ineffective, or if it was previously found that this type of training would not be applicable to the caregiver-client dyad (the answer is no), the subsequent question is whether a caregiver training curriculum could be instituted through telehealth. Factors that might reduce the impact of a caregiver training curriculum (thus making it ineffective) might be related to the caregiver's technological competence, the lack of generalization observed on other caregiver targets, the impact of the crisis situation, and so on. If the answer is no to the utility of a caregiver training protocol or it has been shown to be ineffective at changing the caregiver's behavior, the model recommends an interruption of services with periodic check-ins to either reinstate the Level 5 telehealth model or, if the crisis situation passes, resume services as normal. Practitioners should create documentation that illustrates their process for deciding to interrupt services and the conditions that would qualify for their reinstatement.

\section{Summary}

The purpose of the current article is to provide practitioners with a decision model to help systematize decisions related to the continuity of service during a crisis. Although this model may be useful during the current pandemic of COVID-19, the model was developed to assist in the case of any disaster. Moreover, it calls on behavior analysts to use our analytic and objective assessment skills to make these difficult decisions. Resources such as the Professional and Ethical Compliance Code for Behavior Analysts (BACB, 2014) were used to help create the crisis decision model; however, practitioners should review such information in its entirety.

Cox et al. (in press) outlined a decision model for considering the potential risks associated with both maintaining and interrupting in-person delivery of ABA services. Because the ethical dilemma at hand is complex and these decisions require careful consideration and subtlety, some may mistakenly interpret the message of the Cox article as suggesting that ABA services are nonessential. Instead, Cox et al.'s decision model suggests that delivering ABA services in person may be nonessential for some clients and that a more ethical choice may be to transition to telehealth delivery of essential ABA services. We believe that a decision model that provides additional considerations may therefore be useful. The crisis decision model outlined in the current article provides a structured approach to aiding behavior analysts in assessing when to maintain direct in-person support and the conditions under which risk may be mitigated by designating a caregiver to function as the necessary essential support worker. This represents a subtle but necessary shift in verbiage to ensure that $\mathrm{ABA}$ providers are not redefining established terms in response to a crisis but are instead adjusting assessment and clinical programming procedures to protect continuity of care in the face of decreased essential worker resources.

In addition to the risk assessment considerations outlined by Cox et al., Rodriguez (in press) also recently published a treatment selection model for transitioning direct $\mathrm{ABA}$ services to telehealth during a time of crisis. Rodriguez provides several valuable contributions to the available related research, including structured tools for modifying clinical programming to maintain telehealthbased ABA treatment integrity during a crisis. The recent articles by Cox et al. (in press) and Rodriguez (in press) provide useful information for practitioners transitioning to telehealth, including specific steps for risk assessment and clinical programming. However, there is limited available guidance on how to effectively and efficiently determine individualized needs for ongoing direct essential worker support. The current article adds a multilevel decision model for ABA service provision during a crisis. All three publications support the need for structured telehealth feasibility assessments (for both providers and consumers). Taken together, we hope that practitioners transitioning to telehealth are now better equipped to engage in subtle and complex decision making regarding assessment and treatment recommendations during the current COVID-19 crisis.

Acknowledgements We thank Jesslyn Farros for her assistance in acquiring the literature for this article and Benjamin Heimann for his help with the decision model.

\section{Compliance with Ethical Standards}

Conflict of interest All three authors declare they have no conflict of interest.

Ethical approval This article does not contain any studies with human or nonhuman participants performed by the authors. 


\section{References}

Association of Professional Behavior Analysts. (2020, April 9). Guidelines for practicing applied behavior analysis during the COVID-19 pandemic. Retrieved from https://cdn.ymaws.com/ www.apbahome.net/resource/collection/1FDDBDD2-5CAF4B2A-AB3F-DAE5E72111BF/APBA Guidelines - Practicing During_COVID-19_Pandemic_040920.pdf

Behavior Analyst Certification Board. (2014). Professional and ethical compliance code for behavior analysts. Littleton, CO: Author.

Behavior Analyst Certification Board. (2020, March 29). Ethics guidance for ABA providers during COVID-19 pandemic. Retrieved from https://www.bacb.com/ethics-guidance-for-aba-providers-duringcovid-19-pandemic-2/

The Council of Autism Service Providers. (2020). Organizational guidelines and standards. https://casproviders.org/wp-content/uploads/ 2020/03/CASP_ALS_Booklet_v1_1.3.20.pdf

Cox, D. J., Plavnick, J. B., \& Brodhead, M. T. (in press). A proposed process for risk mitigation during the COVID-19 pandemic. Behavior Analysis in Practice.

Fryling, M. J., Wallace, M. D., \& Yassine, J. N. (2012). Impact of treatment integrity on intervention effectiveness. Journal of Applied
Behavior Analysis, 45, 449-453. https://doi.org/10.1901/jaba. 2012.45-449.

Keenan, M., Dillenburger, K., Röttgers, H. R., Dounavi, K., Jónsdóttir, S. L., Moderato, P., et al. (2015). Autism and ABA: The gulf between North America and Europe. Review Journal of Autism and Developmental Disorders, 2, 167-183. https://doi.org/10.1007/ s40489-014-0045-2.

Pennington, B., Simacek, J., McComas, J., McMaster, K., \& Elmquist, M. (2019). Maintenance and generalization in functional behavior assessment/behavior intervention plan literature. Journal of Behavioral Education, 28, 27-53. https://doi.org/10.1007/s10864018-9299-6.

Pollard, J. S., Karimi, K. A., \& Ficcaglia, M. B. (2017). Ethical considerations in the design and implementation of a telehealth service delivery model. Behavior Analysis: Research and Practice, 17(4), 298-331. https://doi.org/10.1037/bar0000053.

Rodriguez, K. (in press). Maintaining treatment integrity in the face of crisis: A treatment selection model for transitioning direct ABA services to telehealth. Behavior Analysis in Practice.

Publisher's Note Springer Nature remains neutral with regard to jurisdictional claims in published maps and institutional affiliations. 